\title{
Approche Méthodologique D’évaluation De Susceptibilité Des Versants Aux Glissements De Terrain Par Un Modèle Volumique De L'aléa ; Application Au Secteur De Boujibar Al Hoceima (Rif, Maroc)
}

\author{
Allal Labriki \\ Saïd Chakiri \\ Wafae Nouaim \\ Mohamed Allouza \\ Jamil Ezzayani
}

Université Ibn Tofail, Faculté des Sciences, Laboratoire Géosciences des Ressources Naturelles, Kénitra, Maroc

doi: 10.19044/esj.2016.v12n32p206 URL:http://dx.doi.org/10.19044/esj.2016.v12n32p206

\begin{abstract}
This paper presents a new assessment technique of slopes landslide susceptibility, through a geometrical solid model of hazard in reliance on application and comparison of two different approaches (probability and determinist). Here we present a complete methodology for resolving all the technical difficulties encountered during the application of deterministic approach on a catchment basin scale, and to generate an estimating model of hazard not only on the ground surface but also in depth. Indeed, the application of this approach in the area of Boujibar in the south of $\mathrm{Al}$ Hoceima city has allowed to specify that, the deterministic method based on factor of safety (Fs) calculation for hazard evaluation of catchment basin is the most adapted and most suitable method on a local and regional scale.
\end{abstract}

Keywords: Susceptibility, 3D modeling, deterministic assessment, geometric model, voxel

Résumé

Cette étude s'intéresse à une nouvelle technique d'évaluation de la susceptibilité des versants aux glissements de terrain par un modèle solide géométrique de l'aléa en s'appuyant sur l'application et la comparaison de deux approches déférentes (probabiliste et déterministe). Nous présentons ici une méthodologie complète, permettant de résoudre l'ensemble des 
difficultés techniques rencontrées lors de l'application de l'approche déterministe à l'échelle d'un bassin versant, et de produire un modèle d'estimation de l'aléa non seulement à la surface du sol, mais également en profondeur. L'application de cette approche méthodologique au secteur de Boujibar au sud de la ville d'Al Hoceima, a permis de préciser que la méthode déterministe basée sur le calcul du facteur de sécurité (Fs) pour l'évaluation de l'aléa glissements de terrain, est une méthode très précise et plus adaptée à l’échelle locale et régionale.

Mots clés : Susceptibilité, modélisation 3D, évaluation déterministe, modèle géométrique, voxel

\section{Introduction}

Ce travail, s'inscrit dans le cadre d'une tentative et proposition d'un modèle géométrique 3D d'évaluation de l'aléa mouvement de terrain. Il présente des aspects essentiellement pratiques et méthodologiques pour le traitement de données spatiales multisources (géologiques, hydrogéologiques, géotechniques, géophysiques, etc.) avec énumération des variations volumiques des différents paramètres contrôlant les instabilités de terrains (fig. 1). De plus, un exemple de modélisation 3D appliquée au secteur de Boujibar de la ville d'Al Hoceima sera décrit afin de valider et de montrer les étapes de construction de ce modèle. Pour ce faire, nous avons eu recours à des outils de modélisation potentiels pour construire des modèles géométriques 3D, tels que les systèmes d'information géographique (SIG) et les logiciels de conception assistée par ordinateur (CAO). 


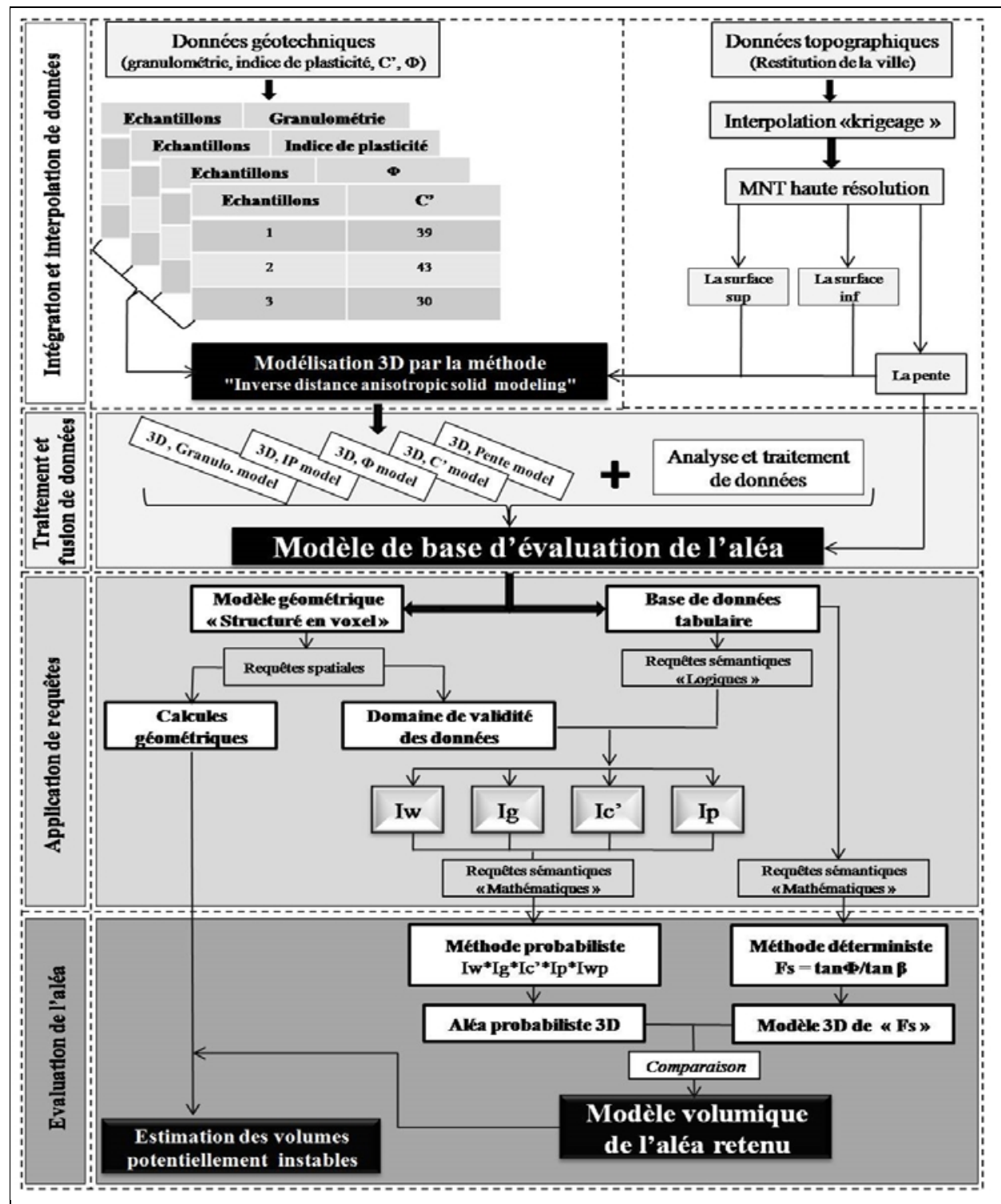

Figure 1 : Approche méthodologique proposée pour la conception d'un modèle volumique de l'aléa

\section{L’approche méthodologique proposée}

Le choix d'une méthode d'évaluation de l'aléa glissements de terrains est lié à l'échelle de la zone étudiée : soit l'échelle locale d'un site instable ou d'un versant, utilisée pour définir le fonctionnement d'un phénomène et simuler des scénarios d'évolution, dans ce cas, les données seront plus nombreuses et plus détaillées; soit l'échelle large du bassin versant pour évaluer et cartographier l'aléa selon des critères morphologiques avec la production d'une cartographie prévisionnelle et 
préventive (Maquaire, 2002). Cependant, quelque soit l'échelle de la zone d'étude, la qualité des résultats dépend avant tout de la qualité des données d'entrée.

Afin de réaliser et proposer un modèle d’évaluation de l'aléa glissement de terrain, l'approche probabiliste fondée sur la simulation statistique des facteurs régissant la stabilité des versants à partir des observations et des données de surface, reste l'un des moyens le plus fiable et le plus utilisé pour repérer les zones soumises aux glissements de terrain (Malatrait et al., 1977, Fares et al., 1994, Margaa, 1994, Mompelat, 1994). L’inconvénient principal de cette approche, est l'évaluation de l'aléa dans un espace bidimensionnel sans aucune prise en considération des variabilités des différents paramètres en fonction de la profondeur (le sous-sol). Pour représenter de façon incontestable ces variations, on peut créer des représentations simplifiées de la réalité qui permettent de formaliser des simulations tridimensionnelles volumiques. Ceci permet d'obtenir un modèle concret qui sert à appliquer des requêtes attributaires et spatiales essentielles pour l'évaluation de l'aléa.

L’approche méthodologique proposée consiste, d'une part, à améliorer les méthodes cartographiques antérieurement élaborées (Fares et al., 1994 ; Margaa, 1994) par la détermination des tendances géométriques et volumiques des différents facteurs d'instabilité dans la quantification de l'aléa au sein d'un espace tridimensionnel et, d'autre part, à obtenir une information sur la stabilité réelle des versants et, par conséquent, l'estimation des volumes mobilisés autour des zones potentiellement instables. Par ce travail, on essaie, à l'échelle d'un bassin versant, de transposer le zonage déterministe de l'aléa fondée sur le calcul de la probabilité à la rupture par un coefficient de sécurité (Fs).

\section{Présentation du secteur de Boujibar}

Le secteur de Boujibar forme l'extension sud du périmètre urbain de la ville d’Al Hoceima (fig. 2). Il est dominé par un climat méditerranéen semi-aride, marqué par des hivers tempérés humides et des étés chauds et secs (Salhi, 2008). Ce climat est conditionné par la présence de la chaîne du Rif qui constitue une barrière interceptant les perturbations en provenance du nord-ouest et du sud-ouest. Le Rif reçoit également des perturbations du nord-est bien que celles-ci soient fréquentes et moins pluvieuses que celles du nord-ouest (Salhi, 2008). Le secteur d’étude est une zone montagneuse marquée par une altimétrie très variable, Cette zone est caractérisée par des pentes variées et des dépôts tendres présentant des mouvements de terrain assez fréquents (Maurer 1968).

Le secteur est occupé au nord par les calcaires du Dévonien de la nappe d'Al Hoceima (fig. 2a et 2d) et au sud et à l'ouest par les reliefs du 
Dhar Imeddiwne formés par les calcaires à silex du Jurassique de la nappe de Jbel Boussicour (Mégard, 1969).

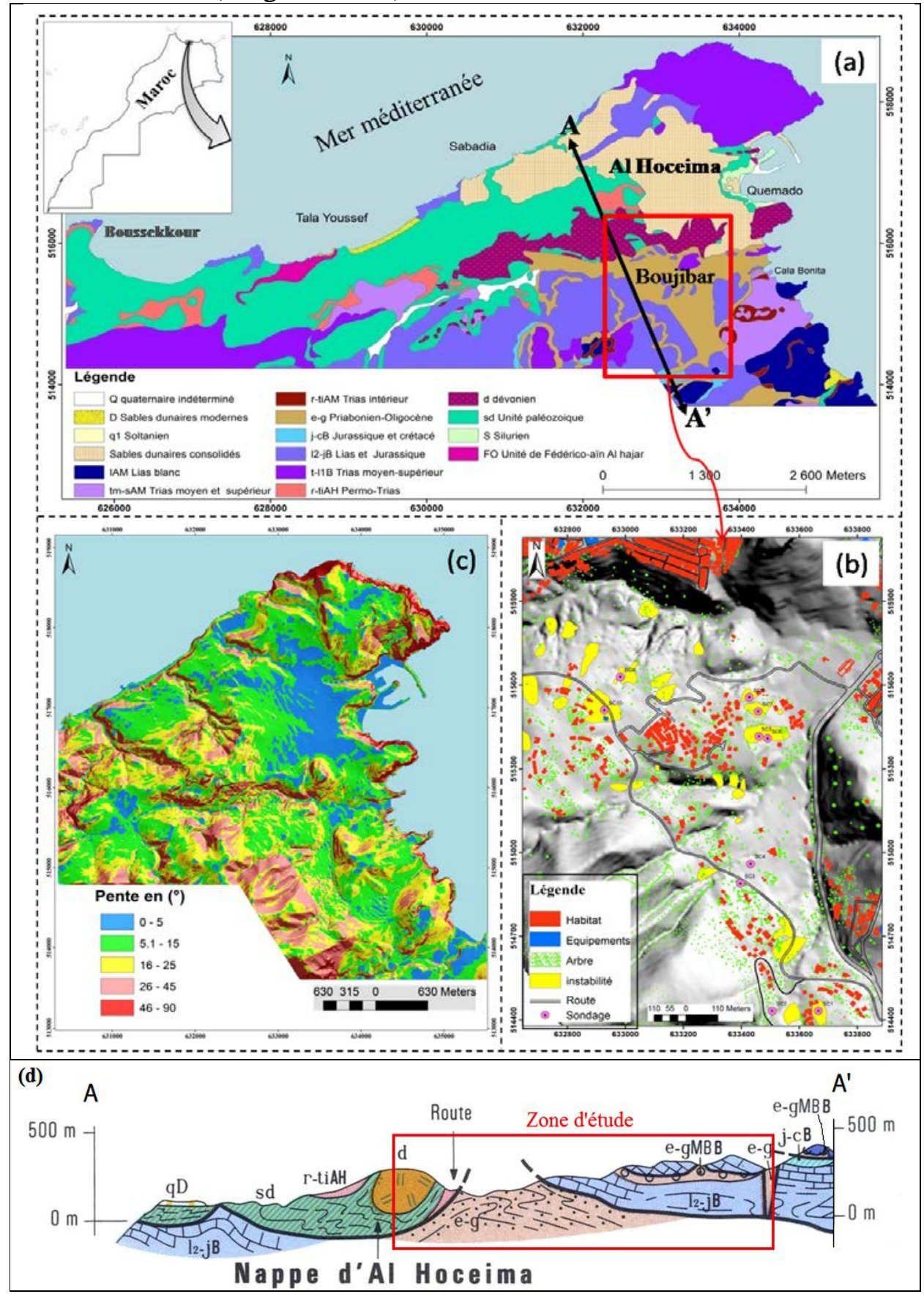

Figure 2 : Cadre géologique et géomorphologique du secteur d'étude.

$\mathrm{a}$ : Situation de la zone d'étude ; $\mathrm{b}$ : Carte détaillée des glissements de terrain dans le secteur de Boujibar; c : carte des pentes de la ville d’Al Hoceima ; d : Coupe géologique au niveau de la zone d'étude (carte géologique d’Al Hocema 1/50000) (légende, fig. (a)). 
Au centre, il est représenté par des faciès variés de marnes, de calcaires et de conglomérats, allant en général de l'Éocène au Miocène inférieur (Mégard, 1963) ; ces dépôt englobent à leur base des éléments de la Dorsale calcaire, alors que leur partie supérieure est marquée par des intercalations instables des dépôts gréso-pélitiques interstratifiés à faciès numioïde (Olivier, 1984 ; Azzouz, 1992). Ces terrains affleurent en particulier au sud de la ville d'El Hoceima et occupent une position structurale inférieure par rapport aux différentes unités structurales de la dorsale externe, de la dorsale interne et des nappes Ghomarides (fig. 2d). Ils se présentent sous forme d'une nappe subhorizontale intercalée entre le massif de Bokoya et les nappes de flyschs tisirènes (Andrieux, 1971).

\section{Essais géotechniques}

Dans le but de déterminer la répartition spatiale volumique des principaux facteurs déclenchant un mouvement de terrain, nous avons eu recour aux données d’investigations géotechniques exécutées en janvier 2014 par l'agence urbaine d'Al Hoceima, dans le cadre du projet de l'étude géologique et géotechnique préliminaire pour le confortement et la stabilisation des glissements de terrain du secteur Boujibar de la ville d'Al Hoceima. Les références spatiales des échantillons, et les résultats d'essais d’identification et des essais mécaniques sont représentés dans les tableaux 1, 2 et 3 .

Tableau 1 : Références spatiales des échantillons analysés

\begin{tabular}{|c|c|c|c|c|c|c|}
\hline \multirow{2}{*}{$\begin{array}{c}\text { Réf. } \\
\text { Echantillon }\end{array}$} & $\begin{array}{c}\text { Sondage } \\
\text { Carotté } \\
\mathrm{N}^{\circ}:\end{array}$ & \multirow{2}{*}{ Nature du sol } & \multicolumn{2}{|c|}{ Coordonnées en $(\mathrm{m})$} & Profondeur \\
\cline { 4 - 6 } & & $\mathrm{x}$ & $\mathrm{y}$ & $\mathrm{z}$ & D’éch. en $(\mathrm{m})$ \\
\hline $001 / 150 / 13$ & $\mathrm{SC} 1$ & Marne jaunâtre & 633666 & 514433 & 168.0 & 8,00 à 9,00 \\
\hline $002 / 150 / 13$ & SC1 & Marne jaunâtre & 633666 & 514433 & 168.0 & 4,00 à 5,00 \\
\hline $003 / 150 / 13$ & SC5 & Marne jaunâtre & 633460 & 515417 & 80.0 & 4,00 à 5,00 \\
\hline $004 / 150 / 13$ & SC5 & Marne jaunâtre & 633460 & 515417 & 80.0 & 6,00 à 7,00 \\
\hline $005 / 150 / 13$ & SC7 & Marne jaunâtre & 633458 & 515506 & 78.0 & 7,00 à 8,00 \\
\hline $006 / 150 / 13$ & SC8 & Marne rosâtre & 633427 & 515556 & 84.0 & 6,00 à 7,00 \\
\hline $007 / 150 / 13$ & SC8 & Marne rosâtre & 633427 & 515556 & 84.0 & 8,00 à 9,00 \\
\hline $008 / 150 / 13$ & SC2 & Marne grise & 633506 & 514433 & 173.0 & 13,00 à 14,00 \\
\hline $009 / 150 / 13$ & SC1 & Marne grise & 633666 & 514433 & 168.0 & 12,00 à 13,00 \\
\hline $010 / 150 / 13$ & SC10 & Marne jaunâtre & 632927 & 515511 & 157.0 & 3,00 à 4,00 \\
\hline $011 / 158 / 13$ & SC10 & Marne jaunâtre & 632927 & 515511 & 157.0 & 6,00 à 7,00 \\
\hline $012 / 158 / 13$ & SC8 & Marne rosâtre & 633427 & 515556 & 84.0 & 5,00 à 6,00 \\
\hline $013 / 158 / 13$ & SC1 & Marne grise & 633666 & 514433 & 168.0 & 14,00 à 15,00 \\
\hline $014 / 158 / 13$ & SC5 & Marne jaunâtre & 633460 & 515417 & 80.0 & 7,00 à 8,00 \\
\hline $015 / 158 / 13$ & SC7 & Marne jaunâtre & 633458 & 515506 & 78.0 & 13,00 à 14,00 \\
\hline
\end{tabular}


Tableau 2 : Résultats des essais d'identification géotechnique

\begin{tabular}{|c|c|c|c|c|c|c|c|}
\hline \multirow{2}{*}{$\begin{array}{c}\text { Réf. } \\
\text { Echantillon }\end{array}$} & \multirow[t]{2}{*}{ Nature du sol } & \multicolumn{2}{|c|}{ Limite d'Atterberg } & \multicolumn{2}{|c|}{ Analyse granulo. } & \multirow{2}{*}{$\begin{array}{l}\text { Densité } \\
\mathrm{T} / \mathrm{m} 3\end{array}$} & \multirow{2}{*}{$\begin{array}{c}\text { Teneur } \\
\text { en eau } \\
(\%)\end{array}$} \\
\hline & & IP \% & WL \% & $>2 \mathrm{~mm}$ & $<0.080 \mathrm{~mm}$ & & \\
\hline $001 / 150 / 13$ & Marne jaunâtre & 23 & 43 & 11.60 & 74.30 & 1.52 & 12.8 \\
\hline $002 / 150 / 13$ & Marne jaunâtre & 27 & 53 & 5.70 & 84.00 & 1.53 & 12.9 \\
\hline $003 / 150 / 13$ & Marne jaunâtre & 23 & 47 & 13.30 & 77.30 & 1.54 & 14.2 \\
\hline $004 / 150 / 13$ & Marne jaunâtre & 28 & 51 & 6.50 & 82.70 & 1.56 & 14.9 \\
\hline $005 / 150 / 13$ & Marne jaunâtre & 25 & 47 & 1.40 & 91.20 & 1.55 & 15.6 \\
\hline $006 / 150 / 13$ & Marne rosâtre & 24 & 48 & 1.20 & 88.20 & 1.59 & 14.3 \\
\hline $007 / 150 / 13$ & Marne rosâtre & 21 & 45 & 3.10 & 85.09 & 1.52 & 13.7 \\
\hline $008 / 150 / 13$ & Marne grise & 27 & 52 & 0.10 & 94.50 & 1.59 & 10.9 \\
\hline 009/150/13 & Marne grise & 26 & 48 & 0.20 & 92.50 & 1.61 & 11.8 \\
\hline 010/150/13 & Marne jaunâtre & 27 & 50 & 1.10 & 90.20 & 1.54 & 12.4 \\
\hline
\end{tabular}

Tableau 3 : Résultats des essais mécaniques

\begin{tabular}{|c|c|c|c|c|c|c|}
\hline \multirow{2}{*}{$\begin{array}{c}\text { Réf. } \\
\text { Echantillon }\end{array}$} & Nature du sol & \multicolumn{2}{|c|}{ Cisaillement rectiligne } & \multicolumn{3}{|c|}{ Essai oedométrique } \\
\cline { 3 - 7 } & & $\mathrm{Cu}(\mathrm{Kpa})$ & $\varphi\left(^{\circ}\right)$ & $\mathrm{Cc}$ & $\mathrm{Cs}$ & Ps (Kpa) \\
\hline $011 / 158 / 13$ & Marne jaunâtre & 16 & 17 & 0.060 & 0.028 & 10 \\
\hline $012 / 158 / 13$ & Marne rosâtre & 8 & 17 & 0.080 & 0.021 & 8 \\
\hline $013 / 158 / 13$ & Marne grise & 52 & 20 & 0.095 & 0.094 & 14 \\
\hline $014 / 158 / 13$ & Marne jaunâtre & 12 & 19 & 0.078 & 0.019 & 11 \\
\hline $015 / 158 / 13$ & Marne jaunâtre & 9 & 16 & 0.097 & 0.017 & 9 \\
\hline
\end{tabular}

\section{Indexation des paramètres retenus}

La cartographie des glissements a permis d'identifier trois principales zones localisées dans les sites Nord-ouest, Est et au Sud du secteur (fig.2b). La rupture de l'équilibre dans ce secteur sous-entend donc la modification d'un paramètre participant à la stabilité ou bien l'adjonction d'un paramètre déstabilisateur. De ce fait, le passage de ce versant de l'état stable à l'état de mouvement résulte de l'action de facteurs de préparation. Par la suite, le mouvement s'initie par action de facteurs de déclenchement. Tout au long de l'étape de génération, les facteurs de prédisposition conditionnent le mouvement en contrôlant sa forme. Les paramètres identifiés dans l'étude de ce secteur sont souvent les mêmes paramètres précités dans les études antérieurs (Margaa, 1994 ; Azzouz et al., 2002).

Tableau 4 : Indexation des paramètres d'entrées pour l'approche probabiliste

\begin{tabular}{|c|c|c|c|c|}
\hline Facteurs & Symboles & Classes & Descriptions & Coefficient \\
\hline \multirow{3}{*}{ Cohésion } & \multirow{2}{*}{$\begin{array}{c}\mathrm{C} \\
\text { En (Kpa) }\end{array}$} & $\mathrm{C}^{\prime}>45$ & $\begin{array}{c}\text { Forte cohésion (matériau } \\
\text { dur). }\end{array}$ & 1 \\
\cline { 3 - 5 } & & $25<\mathrm{C}^{\prime} \leq 45$ & $\begin{array}{c}\text { Cohésion moyenne } \\
\text { (matériau ferme). }\end{array}$ & 2 \\
\cline { 3 - 5 } & & $10<\mathrm{C}^{\prime} \leq 25$ & $\begin{array}{c}\text { Faible cohésion (matériau } \\
\text { tendre). }\end{array}$ & 3 \\
\cline { 3 - 5 } Frottement et pente & \multirow{2}{*}{$\begin{array}{c}\text { et } \beta \\
\text { En }\left(^{\circ}\right)\end{array}$} & $\begin{array}{c}\text { Très faible cohésion } \\
\text { (matériau lâche). }\end{array}$ & 4 \\
\cline { 3 - 5 } & & $\beta<<$ & Domaine de forte stabilité & 1 \\
\cline { 3 - 5 } & & $\beta=\varphi$ & Domaine de faible stabilité & 2 \\
\hline
\end{tabular}




\begin{tabular}{|c|c|c|c|c|}
\hline & & $\beta>\varphi$ & Domaine de rupture & 4 \\
\hline \multirow{3}{*}{ La fraction fine } & \multirow{3}{*}{ FF } & $\leq 12 \%$ & Sols pauvres en fraction fine & 1 \\
\cline { 3 - 5 } & & {$[12 \%-35 \%]$} & $\begin{array}{c}\text { Sols sableux et graveleux } \\
\text { avec la fraction fine }\end{array}$ & 2 \\
\cline { 3 - 5 } & & $\geq 35 \%$ & $\begin{array}{c}\text { Comportement assimilable à } \\
\text { celui de la fraction fine }\end{array}$ & 3 \\
\hline \multirow{3}{*}{$\begin{array}{c}\text { L'indice de } \\
\text { plasticité }\end{array}$} & \multirow{3}{*}{$\begin{array}{c}\text { Ip } \\
\text { En (\%) }\end{array}$} & {$[00-05]$} & Non plastique & 1 \\
\cline { 3 - 5 } & & ] $05-15]$ & Peu plastique & 2 \\
\cline { 3 - 5 } & & $>40$ & Plastique & 3 \\
\cline { 3 - 5 } & & & Très plastique & 4 \\
\hline
\end{tabular}

Les paramètres recensés au départ ne sont pas toujours retenus dans l'analyse et l'évaluation de l'aléa; ainsi, les variables qui ont une contribution nulle ou très faible seront généralement éliminées. De ce fait, le nombre et la qualité des variables retenus sont souvent guidés par les objectifs et la disponibilité des données ; un grand nombre de paramètres ne donnent pas toujours de meilleurs résultats (Gokceoglu, 2001, Remondo et al., 2003).A cet égard, les principaux facteurs d'instabilité pris en compte dans l'évaluation de l'aléa dans le secteur de Boujibar, sont relatifs aux caractéristiques mécaniques (cohésion et frottement) des formations géologiques, à la granulométrie (la fraction fine) et à l'indice de plasticité (IP=WL-WP).Chaque facteur retenu sera codé logiquement, suivant la méthodologie définie par Margaa (1994), et selon la classification géotechnique des comportements mécaniques des sols (tableau 4).

\section{La cohésion}

Elle caractérise les forces de liaison susceptibles de se développer entre les constituants solides et d'induire des variations dans la déformabilité du milieu. L’intégration qualitative de la cohésion est basée sur la nature des matériaux (dur, ferme, tendre, lâche, etc.). Cet aspect reflète le degré d'association des éléments constitutifs en présence d'eau. En effet, lorsque la quantité d'eau est très importante, les particules du sol sont séparées les unes des autres et la cohésion du sol est alors nulle, par contre la présence d'eau à une faible quantité peut donner une certaine cohésion à des sols qui n'en n'ont aucune lorsqu'ils sont secs.

\section{Le frottement interne}

La notion d'angle de frottement interne est une approximation établie par Coulomb (1773) qui permet de représenter les états de contraintes correspondant à la limite entre les domaines stable et instable d'un matériau. Il dépend du type de sol et plus particulièrement de l'émoussé de ses grains ; plus ces derniers sont anguleux, plus l'angle de frottement interne sera élevé. Ainsi, il existe une forte relation entre le frottement interne $(\varphi)$ et la pente du 
talus naturel ( $\beta$ ) (fig. $2 \mathrm{c}, 3$ ) ; cette dernière ne peut dépasser une valeur limite $(\varphi)$ (fig. 3).

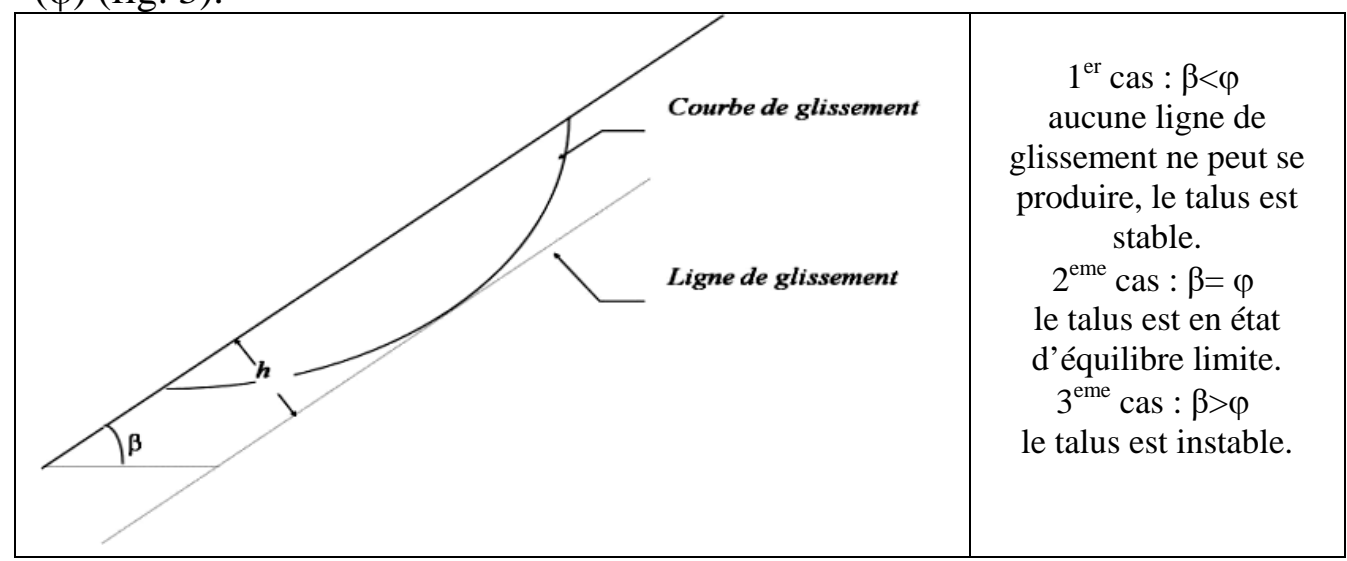

Figure 3 : Etats limites d'apparition d'une ligne de glissement en fonction de la pente $(\beta)$ et de l'angle de frottement interne $(\varphi)$

L’angle $(\varphi)$ dépend de la forme des grains et de la granulométrie :

- $\quad$ grains arrondis et uniformes : $28^{\circ} \leq \varphi \leq 35^{\circ}$

- $\quad$ grains anguleux, granulométrie continue : $35^{\circ} \leq \varphi \leq 45^{\circ}$

\section{La fraction fine}

L'abondance des argiles dans les matériaux du versant a des répercussions sur la stabilité en fonction de leurs caractéristiques géotechniques et minéralogiques (Faleh et al., 2002). Le pourcentage de la fraction fine (tamisât à $80 \mu \mathrm{m}$ ) permet de distinguer entre trois types de sols différents (Norme NF P 11300 et G.T.R) :

- au-delà de 35\%, les sols ont un comportement assimilable à celui de leur fraction fine ;

- $12 \%<80 \mu \mathrm{m} \leq 35 \%$, permet de distinguer les sols sableux et graveleux avec fines ;

- $\quad 80 \mu \mathrm{m} \leq 12 \%$, il s'agit d'un sol permettant de caractériser les sols pauvres en fines.

\section{L'indice de plasticité}

C’est le domaine situé entre les états de liquidité et de plasticité. Il est déterminé par la différence entre la limite de liquidité et la limite de plasticité $\mathrm{IP}=\mathrm{WL}-\mathrm{WP}$. Il permet d'apprécier la faculté d'un matériau à devenir très déformable en absorbant de l'eau, c'est-à-dire, sa plasticité. Un sol dont l'indice IP est grand, est très sensible aux conditions atmosphériques, car plus IP est grand plus le gonflement par humidification de la terre et son retrait par dessiccation seront importants. L’indice de plasticité précise donc 
quatre types de matériaux (non plastique, peu plastique, plastique et très plastique).

\section{Présentation des résultats}

Cette étude dont l'objectif est de comparer deux modèles spatiaux (déterministe et probabiliste) pour évaluer la susceptibilité des versants aux glissements de terrain à l'échelle large (échelle au 1/10000) pour des environnements complexes et accidentés. Ces deux méthodes d'analyse spatiale, implantées dans un SIG, ont été testées sur un site d'étude localisé au sud de la ville d'Al Hoceima (Rif).L'analyse de sensibilité a été réalisé sur un volume théorique de $338220 \mathrm{~km}^{3}$ a une résolution spatiale de $10 \mathrm{~m}$. Ce volume qui comprend 676440 voxels, représente les dimensions des matériaux granulaires valables pour l'évaluation de la susceptibilité aux glissements de terrain jusqu'à une profondeur de $50 \mathrm{~m}$.

\section{Evaluation déterministe}

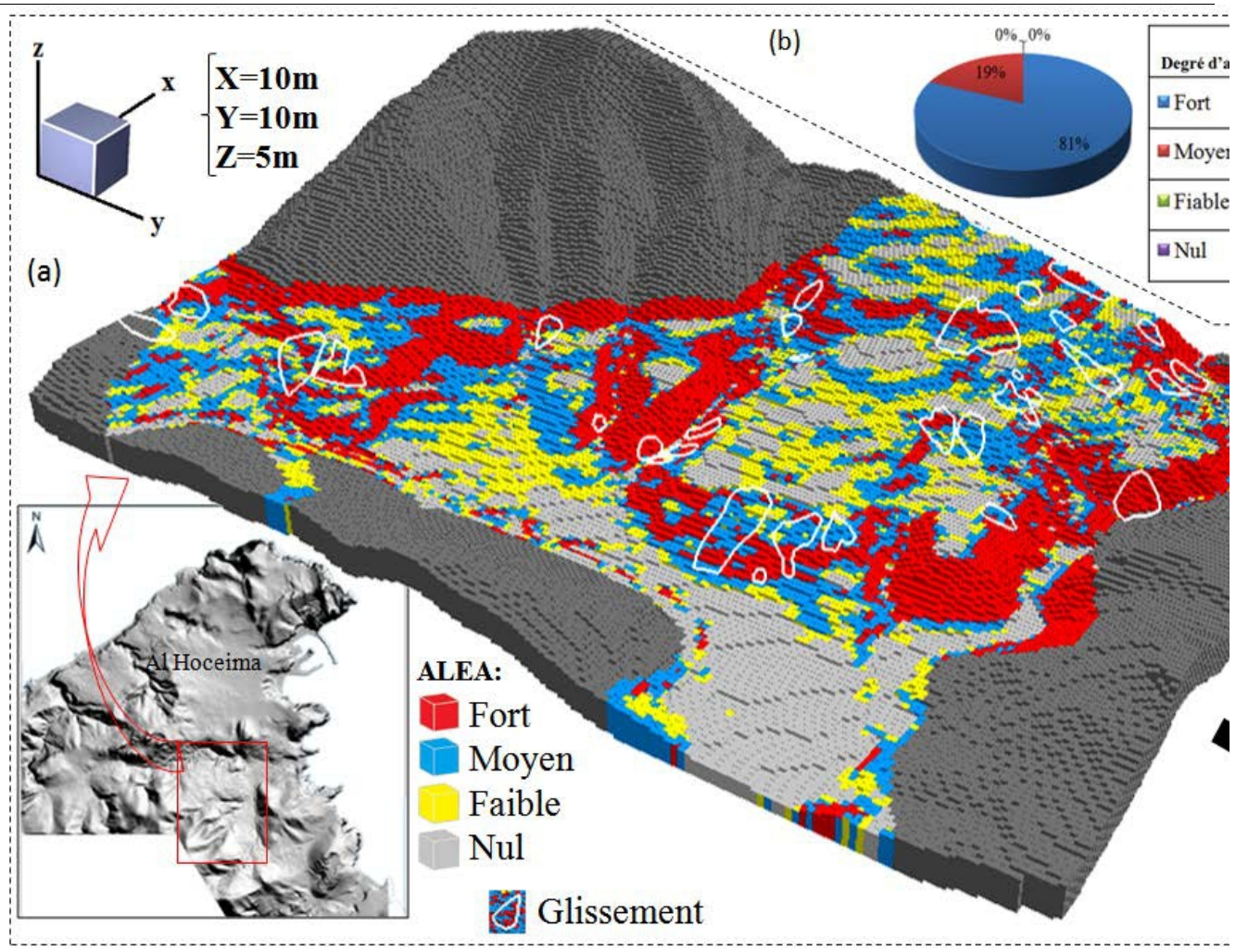

Figure 4 : Le modèle volumique de l'aléa obtenu par l'approche déterministe et distribution des glissements actifs selon le niveau d'aléa.

Le modèle de coefficient de sécurité ne peut être construit sans la connaissance, en tout voxel, des paramètres mécaniques des formations 
géologiques. Puisqu'il est très difficile de connaître en tout point les caractéristiques mécaniques, on doit extrapoler les données existant à chaque voxel du modèle selon la méthode d'interpolation spatiale "inverse distance". Par l'application de l'approche déterministe basée sur le calcul du coefficient de sécurité $(F s=\tan \varphi / \tan \beta)$, on a pu réaliser un modèle volumique en trois dimensions, du coefficient de sécurité. Pour obtenir un zonage significatif de l'aléa lié aux instabilités de terrain, on subdivise les valeurs des coefficients de sécurité en quatre classes (fig. 4) :

- $\quad$ terrains à susceptibilité élevée aux glissements de terrain, pour Fs inférieure à 1 ;

- $\quad$ terrains à susceptibilité moyenne aux glissements de terrain, pour Fs compris entre 1 et 1.5 ;

- $\quad$ terrains à susceptibilité faible aux glissements de terrain, pour Fs compris entre 1.5 et 2 ;

- $\quad$ terrains à susceptibilité nulle aux glissements de terrain, pour Fs supérieur à 2.

L'analyse du résultat de l'évaluation déterministe, montre que, d'une manière générale, l'aléa est bien prononcé au niveau de ce versant (fig. 4) et semble toujours être conditionné par le facteur pente. On remarque de premiers abords que les terrains de susceptibilité moyenne à élevée aux glissements de terrain se retrouvent les plus répandus. Elles représentent un pourcentage de $57 \%$ des terrains de la zone d'étude; les zones à forte instabilité (Fs <1) représentent $28 \%$ de l'ensemble des terrains du secteur. Les terrains à susceptibilité faible à nulle (Fs>1.5) aux glissements de terrain sont largement réduits ; les terrains stables (susceptibilité nulle) ne présentent que $23 \%$ du total de la zone étudiée (fig. 4). 


\section{Evaluation probabiliste}

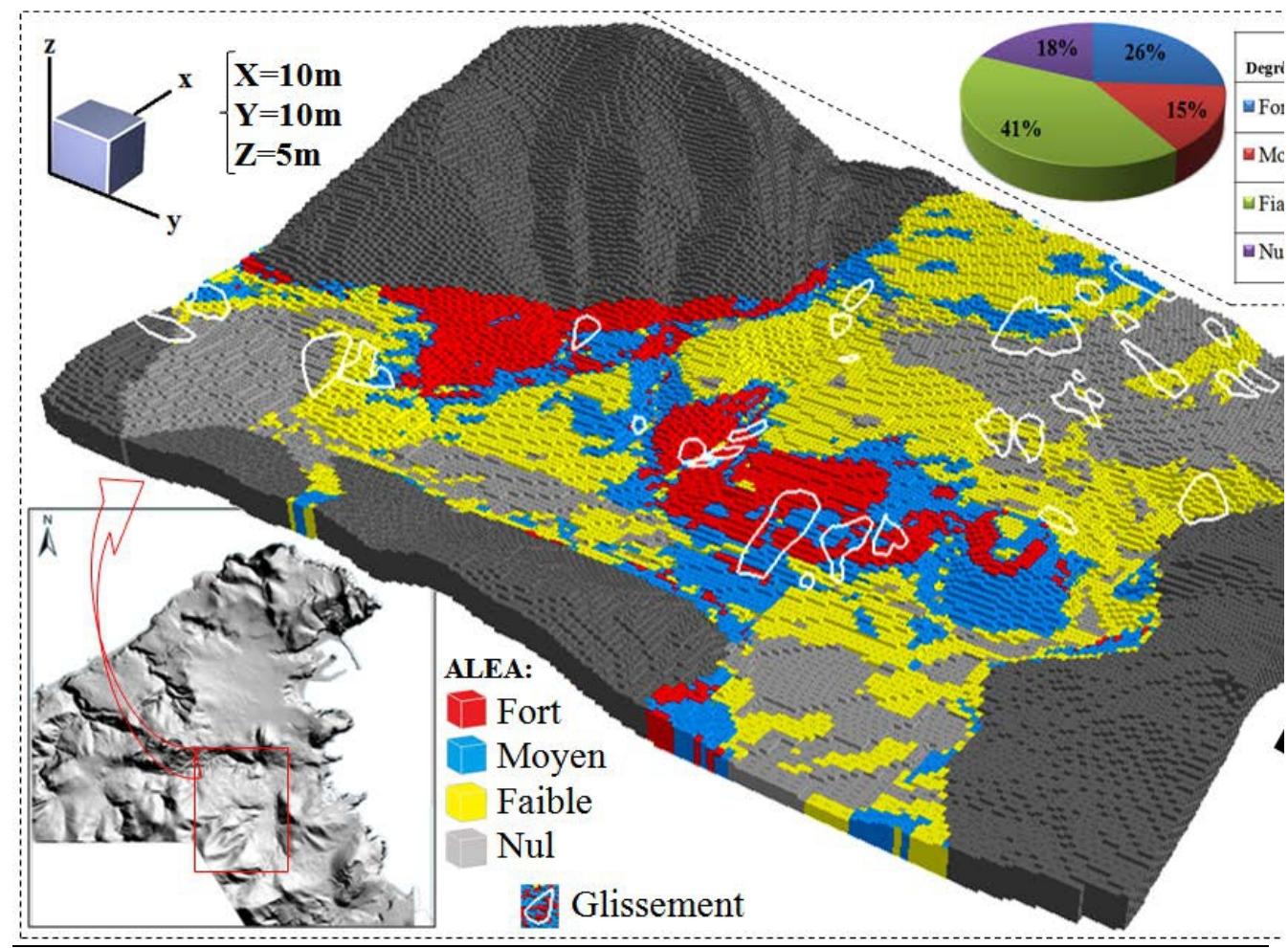

Figure 5 : Le modèle volumique de l’aléa obtenu par l’approche probabiliste et distribution des glissements actifs selon le niveau d'aléa

L'utilisation de la méthode probabiliste comme outil de prédiction spatiale nécessite cependant un travail important de collecte, de mise en forme et de traitement des données de base. Cette méthode est fondée à la fois sur l'expérience de l'expert et sur l'objectivité de la méthode de calcul (Van Westen et al., 2003). L'application de cette méthode indique qu'une combinaison de cinq variables prédictives (la pente, la cohésion, l’indice de plasticité, le frottement interne, et le pourcentage de la fraction fine), permet de produire une valeur théorique de l'aléa dans chaque unité élémentaire de terrain (voxel), en fonction des indices de chaque variable. Le modèle brut obtenu a été reclassé en 4 classes d'aléa (nul, faible, moyen et fort) en termes de susceptibilité aux glissements de terrain (fig.5).

L'examen du résultat obtenu montre que c'est l'aléa faible à nul qui semble le plus répandu dans ce cas ; il couvre $71 \%$ de la zone d'étude, alors que l'étendu spatial de l'aléa moyen à fort est largement réduit ; il occupe seulement29\% de l'ensemble des terrains étudiés, dont la susceptibilité forte aux glissements de terrain ne présente que 10\% (fig. 5). 


\section{Synthèse et discussion}

Les résultats obtenus sont confrontés à la carte des glissements observés sur le terrain. Au total, 27 mouvements de terrain sont retenus ; ils se répartissent régulièrement sur le secteur d’étude selon le degré d’intervention des paramètres contributifs. Ainsi, La comparaison de niveau de corrélation entre l'aléa et les instabilités des deux méthodes, permet de déterminer d'une façon plus réaliste l'approche la plus fiable pour l'évaluation de la susceptibilité à cette échelle. Cette confrontation montre qu'il y à une forte corrélation entre l'aléa et les instabilités dans le cas d’évaluation déterministe (fig. 4). Ainsi, les zones présentant un degré d'aléa fort renferment $81 \%$ des glissements recensés dans ce secteur, les zones restantes se localisent dans les zones qui présentent un niveau moyen de l'aléa (fig. 4). Par contre l'approche probabiliste donne une distribution anormale des glissements de terrain en fonction de classes de susceptibilité (fig. 5) ; les secteurs de faible degré d'aléa se révèlent plus actifs (environ 59\% des glissements de terrain) que les zones montrant une forte susceptibilité aux glissements de terrain; celles-ci renferment seulement $41 \%$ des glissements actifs, dont $26 \%$ se localisent dans les sites à forte susceptibilité aux glissements de terrain (fig. 5).

\section{Conclusion}

Les résultats des deux modèles volumiques de l'aléa sont plus ou moins éloignés. L’objectif était de montrer qu’il est possible de simuler la susceptibilité des versants aux déclanchements des mouvements de terrain par deux approches différentes, afin de déterminer le modèle le plus propice pour l'évaluation de l'aléa à l'échelle large. Les différentes simulations réalisées par les deux approches, montre que l’évaluation déterministe basée sur le calcul du coefficient de sécurité, semble plus valide et plus adaptée à l'échelle de travail considérée (1/10000). Les résultats montrent une très bonne corrélation entre les zones à forte probabilité de rupture $(\mathrm{Fs}<1)$ et les évènements recensés par photo-interprétation et/ou observations de terrain (fig. 4). Par rapport au résultat obtenu par le modèle probabiliste, le modèle physique utilisé ici, tient compte véritablement des processus géologiques et mécaniques des versants. Ce modèle apparait alors plus pratique pour l'estimation de la stabilité réelle des versants. Pour l'approche probabiliste, la comparaison avec la carte des mouvements réels et l'analyse de quelques sites posant problème, montre que certaines zones ne sont pas ou sont faiblement en adéquation avec la réalité du terrain. C'est le cas surtout des secteurs en susceptibilité nul à faible qui présentent $59 \%$ des glissements recensés (fig. 5).

Il ressort alors que l'évaluation déterministe donne une information sur la stabilité réelle du versant. La réalisation du modèle déterministe de 
stabilité n'a pu se faire qu'à partir des mesures systématiques de l'ensemble des paramètres mécaniques ( $\left.\phi^{\prime}, C^{\prime}\right)$ sur la totalité de secteur d'étude. Ce modèle peut ensuite être affiné en ajoutant des paramètres hydrogéologiques et climatiques et le comportement des formations géologiques, suivi d'une observation précise de ce qui pourrait traduire une évolution ultérieur. Ceci pourrait conduire à prescrire certains plans de recommandations et l'élaboration de cartes d'aléa liées aux glissements de terrain pour l'aménagement et aussi pour l'évacuation en cas de risque. Cependant Le zonage obtenu par l'approche probabiliste, reste de portée générale et pourrait servir aussi comme support des études préliminaires au schéma directeur de la ville d'Al Hoceima.

\section{References:}

1. Andrieux, J., 1971. La structure du Rif central. Etude des relations entre la tectonique de compression et les nappes de glissements dans un tronçon de la chaîne alpine. Notes \& Mémoires, Service de la Géologie, Maroc, n² 235, 155 p.

2. Azzouz, O., 1992. Lithostratigraphie et tectonique hercynienne des terrains paléozoïques ghomarides du Massif des Bokoya (Rif Interne, Maroc). Thèse 3ème cycle, Univ. Mohammed V, Fac. Sci. Rabat, 208 p.

3. Azzouz, O., El Fellah, B., Chalouan, A., 2002. Processus de glissement dans le Massif de Bokoya (Rif interne, Maroc) : exemple de Cala Bonita. Bull. Institut sci., Rabat, Sciences de la Terre 24, 3340.

4. Fares, A., Rollet M., Broquet P., 1994. Méthodologie de la cartographie des risques naturels liés aux mouvements de terrain. Revue Française de Géotechnique n 69, p. 63-72.

5. Faleh, A., Sadiki A., Akdim B. \& Haloui B. 2002. Application des analyses minéralogiques et géotechniques des argiles dans l'étude des glissements de terrains : exemples du Prérif central (Maroc septentrional). Pap. Géogr., Univ. Murcia (Espagne), 35, 115-128.

6. Gokceoglu, C., 2001, Discussion on Landslide hazard zonation of the Khorshrostam area, Iran by A. Uromeihy and M.R. Mahdavifar, Bulletin of Engineering Geological Environment, 58, 207-213. Bulletin of Engineering Geological Environment, 60, 79-80.

7. Malatrait, A.M., Letourneur J., Antoine P., 1977. Essai de cartographie systématique des mouvements de terrain dans les Alpes Françaises. Bull. Ass. Int. Geol. De l'Ingénieur, n¹6, pp. 39-46.

8. Maquaire, O., 2002, Aléas géomorphologiques (mouvements de terrain) : processus, fonctionnement, cartographie. Mémoire 
d'habilitation à Diriger des Recherches. Université Louis Pasteur, Strasbourg, $219 \mathrm{p}$.

9. Margaa, Kh., 1994. Essai de cartographie des risques naturels: application à l'aménagement de la région d'Al Hoceïma. Thèse Univ. Franche-Comté, Besançon, France, 196 p.

10. Maurer, G., 1968. Les montagnes du Rif central; étude géomorphologique. Trav. Inst. Sci., série Géol. \& Géogr. phys., 14, Rabat, 499 p.

11. Mégard, F., 1963. La partie orientale du massif des Bokkoya. Note Mém. Serv. Géol. Maroc, 194, P. 123-181.

12. Mégard F. 1969. Etude géologique de la chaîne du Rif : La partie orientale du Massif des Bokoyas. Notes \& Mem. Serv. géol. Maroc, 194. 123-198.

13. Mompelat, P., 1994. Unités cartographiques et évaluation de l'aléa mouvements de terrain en Guadeloupe (Antilles Françaises). Thèse, Paris VI.

14. Olivier, P., 1984. Evolution de la limite entre zones internes et zones externes dans l'arc de Gibraltar. (Maroc-Espagne). Thèse d'Etat, Géologie Université de Toulouse, France, 129p.

15. Remondo, J., Gonzàlez-Diez, A., Dìaz de Teràn, J.R., Cendrero, A., 2003. Landslides susceptibility models utilising spatial data analysis techniques. A case study from the lower Deba Valley, Guipùzcoa (Spain). Natural Hazards, 30, 267-279.

16. Salhi, A., 2009. Géophysique, hydrogéologie et cartographie de la vulnérabilité et du risque de pollution de l'aquifère de Ghis-Nekor ( Al Hoceima, Maroc ). Thèse Univ. Abdelmalek Essaadi, Fac. Sci, Tétouan, Maroc.

17. Van Westen, C.J., Rengers, N., Soeters, R., 2003. Use of geomorphological information in indirect landslide susceptibility assessment. Natural Hazards, 30, 399-419. 